\title{
Application of Graph Theory in Computer Science and Engineering
}

\author{
Rishi Pal Singh \\ Assistant Professor
}

\author{
Vandana \\ Research Scholar
}

\begin{abstract}
Graphs are considered as an excellent modeling tool which is used to model many type of relations amongst any physical situation. Many problems of real world can be represented by graphs. This paper explores different concepts involved in graph theory and their applications in computer science to demonstrate the utility of graph theory. These applications are presented especially to project the idea of graph theory and to demonstrate its objective and importance in computer science engineering.
\end{abstract}

\section{Keywords}

Graphs, connectivity, constraints, graph coloring, graph drawing

\section{INTRODUCTION}

Graph theory is a branch of discrete mathematics. In mathematics and computer science, graph theory is the study of graphs which are mathematical structures used to model pair wise relations between objects. There is wide use of graphs in providing problem solving techniques, because it gives an intuitive manner prior to presenting formal definition. To analyze the graph theory application two problem areas are considered.

\section{1- Classical problem}

2- Problems from applications

the classical problem are defined with the help of the graph theory as connectivity, cuts, paths and flows, coloring problems and theoretical aspect of graph drawing. Whereas problems from application particularly emphasis on experimental research and the implementation of the graph theory algorithms. Graph drawing [1] is a key topic in implementation point of view, because the automatic generation of drawing graph has important applications in key computer science technologies such as data base design, software engineering, circuit designing, network designing and visual interfaces.

\subsection{Graphs}

Graphs provide a convenient way to represent various kinds of mathematical objects. Essentially, any graph is made up of two sets:

1- A set of vertices

2- A set of edges.

Depending on the particular situation, restrictions are imposed on the type of edges we allow. For some problems directed edges are applied and for other problem undirected edges are applied from one vertex to other. So graphs give us many techniques and flexibility while defining and solving a real life problem. Graphs has many features, some of them are:

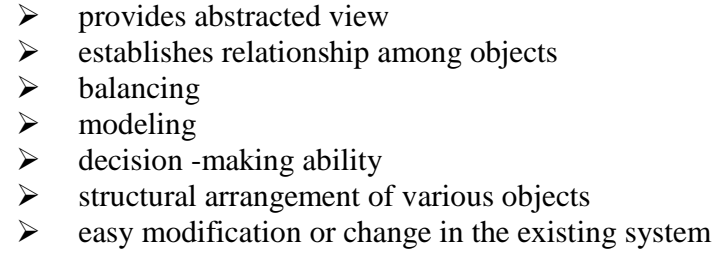

\section{APPLICATION IN COMPUTER SCIENCE}

\subsection{Data base designing}

In data base designing graphs are used as graph data bases [2]. Graph database uses graph representation with nodes, edges, and properties to represent and store data. This graph structure has key role in designing database, because it gives fast implementation process using different functionality and properties of graph structure .Graph database uses as:

\footnotetext{
> Storage system that provides index free adjacency

$>$ Analyzing tool for interconnection

$>$ Powerful tool for graph like-query

> Graph databases are often faster for associative data sets that map more directly to the structure of object-oriented applications.
}

\subsection{Software engineering}

Graph has many applications in software engineering. For example: during Requirements Specification, Data Flow diagrams are used where vertices represent transformations and edges represents the data flows. During Design phase, graphical design is used for describing relations among modules; while during Testing, the control flow of a program associated with McCabe's complexity measure which employs directed graphs for addressing the sequence of executed instructions and etc. Even Software Process Management has also applications of network diagrams which involves graph algorithms.

\subsection{Network system}

Graph theory has wide application in the field of networking. To analyze the graph theory application in networking two areas are considered: graph based representation and network theory. Graph based representation has many advantages such as it gives different point of view; it makes problem much easier and provide more accurate definition. Whereas network theory provide a set of techniques for analyzing a graph and applying network theory using a graph representation. The term graph and network are equal. Both refer to a type of structure in which there exists vertices (i.e. nodes, dots) and edges (i.e. links, lines). There are numerous types of graphs and networks which yield more or less structure. These two 
terms can be differentiating on the basis of their utility. the term graph is used in mathematics whereas the term network is used in physics.

\subsubsection{Purpose of a Graph in mathematics}

Mathematicians are concerned with the abstract structure of a graph.

$>$ Mathematician's define operations to analyze and manipulate graphs.

$>$ They develop theorems based upon structural axioms.

\subsubsection{Purpose of a Network in physics}

$>$ Physicists are concerned with modeling real-world structures with networks.

$>$ Physicist's define algorithms that compress the information in a network to more simple values (e.g. statistical analysis).

Graph theoretic concepts are applied in several fundamental issues in network such as connectivity, data gathering, routing, mobility, energy efficiency, topology control, traffic analysis, finding shortest path and load balancing [11].

\subsection{Computer hardware}

In computer hardware graph theory concepts are used to model the limitation of the physical layer.

Graph theory concepts are used in hardware world to provide:

$>$ Register allocation by graph coloring

$>$ Representation of instruction sequences by graphs by adjacency matrix

$>$ In instruction parallel processing

$>$ Process of allocation scheduling[3]

\subsection{Data structure}

Data may be organized many different ways. The logical or mathematical model of a particular organization of data is called a "data structure". The choice of data model depends on two considerations:

$>$ It must be rich enough in structure to mirror actual relationship of data in real world.

$>$ The structure should be simple enough that one can effectively process data when necessary.

These two considerations is fulfilled by the graph theoretical concepts. Arbitrary relation among data can also be represented by a graph and its matrices, operations performed on these metrics are further useful for deriving relations and data association and is useful in order to understand how these data may be stored in memory [4][5].

\subsection{Image processing}

Image Analysis is the methodology by which information from images is extracted. Image analysis is mainly performed on digital image processing techniques. The image processing techniques can be improved using a graph theoretic approach. The applications of graphs in image processing are: to find edge boundaries using graph search algorithms in segmentation.

$>$ To calculate he alignment of the picture

$>$ Finding mathematical constraints such as entropy by using minimum spanning tree.

$>$ Finding distance transforms of the pixels and calculates the distance between the interior pixels by using shortest path algorithms.

\subsection{Data mining}

Graph mining is the main application area of graph theory in data mining. Graph mining represents the relational aspect of data. There are five theoretical based approaches of graph based data mining. They are sub graph categories, sub graph isomorphism, graph invariants, mining measures and solution methods.

\subsection{Operating system}

A graph is a data structure of finite set of pairs, called edges or vertices. Many practical problems can be solved with the help of graph in the field of operating system such as job scheduling and resource allocation problems. For example graph coloring concept can be applied in job scheduling problems of CPU, jobs are assumed as vertices of the graph and there will be an edge between two jobs that cannot be executed simultaneously and there will be one to one relationship between feasible scheduling of graphs [10].

\subsubsection{Graph purpose in operating system}

$>$ System processes are represented in graph form.

$>$ Graph extraction techniques are used in event tracing.

$>$ Excellent testing tool in performance evaluation because of easy validation and modification.

\subsection{Website designing}

Website designing can be modeled as a graph, where the web pages are represented by vertices and the hyper links between them are represented by edges in the graph. This concept is known as web graph. Which discover the interesting information? Other application areas of graphs are in web community. Where the vertices represent classes of objects, and each vertex representing one type of objects, and each vertex representing a type of object is connected to every vertex representing other kind of objects. In graph theory such a graph is called a complete bipartite graph. There are many advantages of using graph representation in website development such as:

$>$ Searching and community discovery.

> Graph representation (directed graph) in web site utility evaluation and link structure.

$>$ Finding all connected component and provide easy detection. 
4. TABLE 1: Application of Graph Theory in Computer Science and Engineering

\begin{tabular}{|c|c|c|c|}
\hline $\begin{array}{l}\text { Application } \\
\text { field }\end{array}$ & $\begin{array}{l}\text { Property and concepts } \\
\text { of graph theory }\end{array}$ & Applications area & Uses \\
\hline $\begin{array}{l}\text { Database } \\
\text { designing }\end{array}$ & Index-free adjacency & Graph database & $\begin{array}{l}\text { Analyzing interconnection, Direct mapping and } \\
\text { Natural scaling to large data sets. }\end{array}$ \\
\hline $\begin{array}{l}\text { Software } \\
\text { engineering }\end{array}$ & $\begin{array}{l}\text { Connectivity and Data flow, } \\
\text { Directed graph, } \\
\text { and cyclomatic complexity }\end{array}$ & $\begin{array}{l}\text { DFD, Control flow } \\
\text { graph, ER-diagram, } \\
\text { process sequencing and } \\
\text { software quality[12] }\end{array}$ & $\begin{array}{l}\text { Transformations, Capturing requirements, } \\
\text { Describing relations among modules, Designing } \\
\text { system and In testing process. }\end{array}$ \\
\hline $\begin{array}{l}\text { Computer } \\
\text { hardware }\end{array}$ & $\begin{array}{l}\text { Graph coloring and Matrix } \\
\text { representation }\end{array}$ & $\begin{array}{l}\text { Coloring algorithms } \\
\text { Fine-grain parallelism } \\
\text { analysis, Data } \\
\text { dependence matrix }\end{array}$ & $\begin{array}{l}\text { Compilers uses graph coloring algorithms for } \\
\text { Register allocation to variables, Calculate } \\
\text { parallelism degree, Very useful in analytical } \\
\text { modeling[3], Addressing the sequence of } \\
\text { instruction execution, Resource allocation and } \\
\text { Economizing the memory space(file } \\
\text { organization). }\end{array}$ \\
\hline Network design & $\begin{array}{l}\text { Connectivity, } \\
\text { Traversing, Adjacency, Vertex } \\
\text { cover algorithms and } \\
\text { Different graph } \\
\text { representation }\end{array}$ & $\begin{array}{l}\text { Topological control } \\
\text { and Weighted graph, } \\
\text { Butterfly network and } \\
\text { 2-D array }\end{array}$ & $\begin{array}{l}\text { Finding shortest path, Searching and } \\
\text { Arrangement of nodes in network designing, } \\
\text { Modeling communication networks [7], Traffic } \\
\text { analysis and in Network security. }\end{array}$ \\
\hline Data Structure & $\begin{array}{l}\text { Directed graphs, Matrices } \\
\text { and Matrices operation }\end{array}$ & $\begin{array}{l}\text { Array, Tree, Linked } \\
\text { list, Pointers, Stacks, } \\
\text { and Queues }\end{array}$ & $\begin{array}{l}\text { Efficient organization of data, Finding minimum } \\
\text { cost tree, Minimizing data retrieval times, } \\
\text { Minimizing page swapping in data structure } \\
\text { paging system and Provides link structure in } \\
\text { websites }\end{array}$ \\
\hline Image processing & $\begin{array}{l}\text { Edge connectivity, Regions, } \\
\text { Spanning tree }\end{array}$ & $\begin{array}{l}\text { Edge boundaries, } \\
\text { Entropy, Shortest path } \\
\text { algorithms and Search } \\
\text { algorithms }\end{array}$ & $\begin{array}{l}\text { Segmentation and registration, Distance } \\
\text { transform and Center line extraction }\end{array}$ \\
\hline Data mining & Sub graphs, Isomorphism & Graph mining & Sub structure matching, Reducing search space. \\
\hline Operation system & $\begin{array}{l}\text { Graph coloring, Directed } \\
\text { graph }\end{array}$ & $\begin{array}{l}\text { Job scheduling } \\
\text { problems and } \\
\text { Simultaneous } \\
\text { execution of job }\end{array}$ & $\begin{array}{l}\text { Provide feasible solution to job scheduling and } \\
\text { Efficient resource(processor) allocation in } \\
\text { solving simultaneous job execution problems.[8] } \\
\text { and In process representation }\end{array}$ \\
\hline Web site designing & $\begin{array}{l}\text { Directed graph, Undirected } \\
\text { graph, In degree and out } \\
\text { degree, } \\
\text { Search algorithms and } \\
\text { Bipartite graph }\end{array}$ & $\begin{array}{l}\text { Web graph, Web pages } \\
\text { and Hyperlinks }\end{array}$ & $\begin{array}{l}\text { Community discovery, Searching } \\
\text { and Website evaluation }\end{array}$ \\
\hline
\end{tabular}

\section{CONCLUSION}

This paper is designed to benefit the students of computer science to gain depth knowledge on graph theory and its relevance with other subjects like operating systems, Networks, Databases, software engineering etc. this paper focused on the various applications of major graph theory that have relevance to the field of computer science and applications. 


\section{REFERENCES}

[1] Graph algorithms and applications: special issue by Nishizeki, Takao; Tamassia, Roberto; Wagner, Dorothea. - Berlin: Springer, 2000. - (Algorithmica: 26. 2000, 1)

[2] Designing Graph Database Models from Existing Relational Databases by Subhrajyoti Bordoloi Bichitra Kalita Dept. Of Computer Applications Dept. Of Computer Applications Assam Engg. College, Guwahati, Assam Engineering. College, Guwahati, Assam.(International Journal of Computer Applications (0975 -8887), Volume 74, no-1,July 2013)

[3] On Applying Graph Theory to ILP Analysis by Raúl Durán, Rafael Rico Department of Computer Engineering, Universidad de Alcalá, Spain(2005)

[4] 4-graph application to data structures by V.manjula (2012)

[5] On the application of graph theory to computer data structure by R.Williams.

[6] A genetic framework for the application of graph theory to image processing by sonali barua (2007)

[7] Application of graph theory in communication networks by suman deswal and Anita singhrova, International
Journal of Application or Innovation in Engineering \& Management (IJAIEM), Volume 1, Issue 2, and October 2012

[8] The role of data structure in multiple disciples in computer science: A Review by N.Sobhna Rani and Suman S.P, International Journal of Scientific \& Engineering Research, Volume 4, Issue 7, July-2013

[9] Graph Databases: Adrian Silvescu, Doina Caragea, Anna Atramentov Artificial Intelligence Research Laboratory Department of Computer Science Iowa State University Ames, Iowa 50011

[10] Applications of Graph Coloring in Modern Computer science by Shamim Ahmed (ijcit, issn 2078-5828 (print), issn 2218-5224 (online), volume 03, issue 02, manuscript code: 130101(2012)

[11] Applications of graph theory in computer science an overview by S.G.Shirinivas, S.Vetrivel and Dr. N.M.Elango (International Journal of Engineering Science and Technology, Vol. 2(9), 2010, 4610-4621)

[12] Graph theory application in developing software test strategies for networking system by Vladimir. V. Riabov (2007) 